\title{
Association between concentration of melatonin, and lipoproteins, LPO, hsCRP, NTproBNP in chronic heart failure patients
}

\author{
Elzbieta Kimak ${ }^{1 *}$, Grzegorz Dzida ${ }^{2}$, Dariusz Duma ${ }^{1}$, Andrzej Prystupa ${ }^{2}$, \\ Magdalena Halabis ${ }^{1}$, Aleksandra KimaK ${ }^{3}$, Bartosz Zieba ${ }^{1}$, \\ IwONa Kaznowska-Bystryk ${ }^{1}$, Agnieszka Kowalska ${ }^{1}$
}

\author{
${ }^{1}$ Department of Laboratory Diagnostics, Medical University of Lublin, Chodzki 1, 20-093 Lublin, Poland \\ ${ }^{2}$ Department of Internal Diseases, Medical University of Lublin \\ ${ }^{3}$ Chair and Department of Conservative Dentistry with Endodontics, Medical University of Lublin
}

\begin{tabular}{l}
\hline ARTICLE INFO \\
\hline Received 23 October 2014 \\
Accepted 4 November 2014 \\
\hline
\end{tabular}

Keywords:

lipoproteins,

hsCRP,

melatonin,

oxidative stress,

chronic heart failure.

\begin{abstract}
The aim of the study was to examine concentrations and relationships between melatonin levels assessed at 0:200 hrs and 0:700 hrs, lipid hydroperoxide (LPO) assessed at 0:200 hrs and 0:700 hrs, and apolipoprotein (apo)AI, apoAII, apoB, high sensitivity C-reactive protein (hsCRP) and NT-proBNP, in 27 patients with chronic heart failure (CHF) (17 patients - with NYHA class II and 10 - with NYHA class III). In the study, Lipoproteins apoAI, apoAII, apoB, high sensitivity C-reactive protein (hsCRP) levels were determined by way of immunonephelometric methods, serum melatonin concentration was measured by using a competitive enzyme immunoassay technique, while serum LPO concentration was measured by using Cayman's Lipid Hydroperoxide Assay Kit. In the study, CHF patients without acute inflammatory response demonstrated a decreased concentration of high density lipoprotein cholesterol (HDL-C), apoAI, apoAII levels, but an increased concentration of NT-proBNP, hsCRP and LPO at night, and LPO at daytime; however, the concentration of LPO at 0:700 was lower than at 0:200. Pearson's correlation test and multiple ridge stepwise regression showed that melatonin administered at night exerts an effect on the composition of apoAI and apoAII of HDL particles, and induces decreased LPO at 0:700, but has no effect upon NT-proBNP levels in patients with NYHA class II. However, in patients with NYHA class III, melatonin administered at night induces an increase in the content of apoAII and apoAI, which further decreases hsCRP, and this, together with the administered melatonin, brings about daytime decreases in NT-proBNP and hsCRP levels. The results indicated that the content of apoAII and apoAI in HDL particles and melatonin demonstrate an anti-oxidative and anti-inflammatory effect, and together, have a cardio-protective effect on patients with advanced CHF. Hence, the results support melatonin being a cardio-protective agent. These relationships, however, need to be confirmed in further studies.
\end{abstract}

\section{INTRODUCTION}

Melatonin is produced in the pineal gland and is released to circulation at night-time. Age-related deterioration in the immune system, which is referred to as immunosenescence, contributes to an increased susceptibility to infection

\footnotetext{
Corresponding author

e-mail: elzbieta.kimak@wp.pl,

tel/fax: (+48 81) 7423813
}

diseases, autoimmunity, dyslipidemia, hypertension, chronic heart disease and cancer in elderly patients [1,7]. Melatonin also plays a crucial role in the organism as it is an antiinflammatory, anti-oxidant and anti-hypertensive agent, and may also be useful in the reduction of cardiac hypertrophy in some situations, thereby limiting the frequency of heart failure [20]. The findings from human and animal studies support the consideration of melatonin as a cardio-protective 
agent $[5,20]$, as there is a general agreement that the reactive oxygen species (ROS) play an important role in the pathogenesis of cardiac ischemia/reperfusion injury [4,18], and Melatonin efficiently interacts with several reactive oxygen species [18]. What is more, in normoglycemic mothers, melatonin increases the ability of colostrum to protect against Escherichia coli and other infections. However, in diabetic mothers, the effects of melatonin are likely limited to antiinflammatory processes, with low superoxidase release and bactericidal activity [15].

Unfortunately, there is no information about the association of melatonin with lipoproteins level, nor is there information on its relation between the composition and metabolism of HDL particles in chronic heart failure (CHF) patients with NYHA class II and III.

\section{AIM}

The aim of the presented study was to examine the relationship between the concentration of melatonin (assessed at 0:200 hrs and 0:700 hrs) and lipid hydroperoxide (LPO) (assessed at 0:200 hrs and 0:700 hrs), and apolipoprotein (apo)AI, apoAII, apoB, hsCRP and NT-proBNP in chronic heart failure $(\mathrm{CHF})$ patients with NYHA class II and III.

\section{MATERIAL AND METHODS}

A total of 27 patients who were diagnosed with chronic heart failure (CHF) according to ESC criteria, aged 71 (51-88) years, and 15 apparently normolipidemic healthy individuals as control, were recruited for the study. The CHF patients had undergone treatment in the Department of Internal Disease of the Medical University in Lublin. These patients did not have myocardial infraction in the last 3 months, nor unstable angina pectoris, infections, malignancies or neuropsychiatric disorders [6]. The patients were placed into 3 groups: all patients, patients with NYHA class II $(n=17)$, and NYHA class III $(n=10)$ [6]; however, CHF patients with higher CRP were excluded from the studied groups. The study was conducted in accordance with the guidelines of the Ethics Committee of the Medical University in Lublin, Poland.

Routine laboratory parameters were obtained in serum after a 14-h overnight fasting. Blood was taken from a vein into commercial test tubes. The serum was immediately separated and stored in aliquots at $-80^{\circ} \mathrm{C}$ until use. Using routine laboratory methods, the level of creatinine and NT-proBNP, and lipids were measured on the Cobas Integra analyzer (Germany). Low density lipoprotein cholesterol (LDL-C) was calculated according to the Friedewald formula [8], while Non-HDL-C was calculated as total cholesterol (TC) minus HDL-C. Serum melatonin concentration at 0:200 hrs and 0:700 hrs was measured using the competitive enzyme immunoassay technique (USCN Life Science Inc., Wuhan, China; Polish distributor - Immuniq). As this assay employs the competitive inhibition enzyme immunoassay technique, an inverse correlation between melatonin concentration in the sample and the assay signal intensity is measured.

ApoAI, apoAII, apoB and high sensitivity C-reactive protein (hsCRP) levels were determined by way of immunonephelometric methods, using the Health Care Diagnostic Product (Siemens GmbH, Germany), on a Dade Behring nephelometer BNII System (Germany). Serum LPO concentration at 0:200 hrs and 0:700 hrs was measured using Cayman's Lipid Hydroperoxide Assay Kit (LPO). This measures the hydroperoxides directly, utilizing the redox reactions with ferrous ions. Hydroperoxides are highly unstable and react readily with ferrous ions to produce ferric ions. The resulting ferric ions are detected by using thiocyanate ion as the chromogen.

Statistical analysis. The data were expressed as medians and minimum-maximum. Shapiro-Wilk's test was used to investigate whether the variables had a normal distribution. The variables with skewed distribution were log 10 transformed. However, the values in the Tables are presented as non-transformed data. The statistical analysis of the obtained results was performed using the nonparametric Kruskal-Wallis test for comparison of patient groups and the reference group. The nonparametric Wilcoxon's t-paired test was used to compare the concentration of LPO at 0:200 hrs and 0:700 hrs in all CHF patients. The relation between the concentration of apoAI, apoAII, apoB, NT-proBNP, hsCRP, LPO at 0:200 hrs and 0:700 hrs, and melatonin at 0:200 hrs and 0:700 hrs was examined by Pearson's correlation analysis. Multiple ridge stepwise forward regression analysis was then used to investigate the relationship between melatonin at 0:200 hrs and 0:700 hrs as an independent variable and apoAI, apoAII, apoB, NT-proBNP, hsCRP, LPO 0:200 hrs and 0:700 hrs concentrations as dependent variables, and for each of the dependent variables, parameters were calculated according to the equation: $y=\beta 0+\beta 1 \times 1+\beta 2 \times 2+\ldots+\beta n \times n$. In the model of multiple regression analysis, high correlations between predictor variables result in inadequate regression coefficients. In such cases, multiple ridge stepwise forward regression analysis improves the accuracy of the model. The relationship between the dependent variables is expressed by the coefficient of multiple regression $(\beta)$, which provides information about the relationship between the dependent and the independent variables. A statistical analysis was performed using the STATISTICA program (StatSoft, Krakow, Poland).

\section{RESULTS}

Concentration of serum lipids, apoAI, apoAII, apoB, NTproBNP, and hsCRP, LPO at 0:200 hrs, LPO at 0:700 hrs, and melatonin at 0:200 hrs and 0:700 hrs, as well as the lipid and lipoprotein ratios are shown in Table 1 . The results of the presented study show that CHF patients had different dyslipidemia, dyslipoproteinemia and atherogenic lipid and lipoprotein ratios and significantly increased concentration of NT-proBNP, hsCRP and LPO at 0:200 hrs and at 0:700 hrs, but not a different level of melatonin at 0:200 hrs and 0:700 hrs, compared to the reference group. However, the nonparametric Wilcoxon's t-paired test demonstrated $(\mathrm{p}<0.05)$ that the concentration of LPO at 0:700 hrs was lower than LPO level at 0:200 hrs in all studied groups. Moreover, patients with NYHA class III had lower lipids, apoAI, apoAII level, and higher hsCRP and NT-proBNP levels than did patients with NYHA class II (Tab. 1). 
Table 1. Concentration of lipid, apoAI, apoAII, apoB, lipid and lipoprotein ratios, hsCRP, NT-proBNP, and LPO 0:200 hrs, LPO 0:700 hrs, melatonin 0:200 hrs, melatonin 0:700 hrs, in a control group, and CHF - in all patients and in patients with NYHA class II and NYHA class III, median, (min-max)

\begin{tabular}{|c|c|c|c|c|}
\hline & $\begin{array}{c}\text { Control group } \\
n=15\end{array}$ & $\begin{array}{c}\text { all CRF } \\
\text { patients } \\
n=27\end{array}$ & $\begin{array}{c}\text { NYHA II } \\
n-17\end{array}$ & $\begin{array}{c}\text { NYHA III } \\
n=10\end{array}$ \\
\hline TG mmol/L & $\begin{array}{c}1.04 \\
(0.87-2.49)\end{array}$ & $\begin{array}{c}1.07 \\
(0.48-3.75)\end{array}$ & $\begin{array}{c}1.11 \\
(0.48-3.75)\end{array}$ & $\begin{array}{c}1.04 \\
(0.48-1.6)\end{array}$ \\
\hline TC mmol/L & $\begin{array}{c}4.63 \\
(3.00-5.52) \\
\end{array}$ & $\begin{array}{c}3.62 \\
(1.99-6.11)^{a}\end{array}$ & $\begin{array}{c}3.70 \\
(2.46-6.11)^{\mathrm{a}} \\
\end{array}$ & $\begin{array}{c}3.34 \\
(1.99-4.43)^{\mathrm{a}}\end{array}$ \\
\hline LDL-C mmol/L & $\begin{array}{c}2.18 \\
(1.38-3.52) \\
\end{array}$ & $\begin{array}{c}1.95 \\
(0.93-4.43) \\
\end{array}$ & $\begin{array}{c}2.18 \\
(1.35-3.26) \\
\end{array}$ & $\begin{array}{c}1.74 \\
(0.93-4.43) \\
\end{array}$ \\
\hline HDL-C mmol/L & $\begin{array}{c}1.39 \\
(1.26-1.52) \\
\end{array}$ & $\begin{array}{c}0.83 \\
(0.23-1.53)^{\mathrm{a}}\end{array}$ & $\begin{array}{c}0.88 \\
(0.67-1.53) a \\
\end{array}$ & $\begin{array}{c}0.75 \\
(0.23-1.06)^{a}\end{array}$ \\
\hline Non-HDL-C mmol/L & $\begin{array}{c}3.24 \\
(1.99-4.64) \\
\end{array}$ & $\begin{array}{c}2.67 \\
(1.58-5.49)\end{array}$ & $\begin{array}{c}2.66 \\
(1.63-5.49) \\
\end{array}$ & $\begin{array}{c}2.49 \\
(1.58-2.94) \\
\end{array}$ \\
\hline ApoA-I mg/L & $\begin{array}{c}1570 \\
(1420-1650) \\
\end{array}$ & $\begin{array}{c}970 \\
(470-1200)^{a}\end{array}$ & $\begin{array}{c}1060 \\
(760-1200)^{a}\end{array}$ & $\begin{array}{c}910 \\
(470-990)^{a} \\
\end{array}$ \\
\hline ApoB mg/L & $\begin{array}{c}750 \\
(680-1140)\end{array}$ & $\begin{array}{c}650 \\
(480-1240)\end{array}$ & $\begin{array}{c}710 \\
(500-1240)\end{array}$ & $\begin{array}{c}620 \\
(480-760)\end{array}$ \\
\hline ApoAII mg/L & $\begin{array}{c}330 \\
(300-360) \\
\end{array}$ & $\begin{array}{c}200 \\
(130-330)^{\mathrm{a}}\end{array}$ & $\begin{array}{c}220 \\
(190-330)^{a}\end{array}$ & $\begin{array}{c}150 \\
(130-200)^{\mathrm{a}}\end{array}$ \\
\hline TC/HDL-C & $\begin{array}{c}3.31 \\
(3.15-6.67)\end{array}$ & $\begin{array}{c}4.37 \\
(1.99-10.1)^{\mathrm{a}}\end{array}$ & $\begin{array}{c}4.20 \\
(1.99-6.94)^{a}\end{array}$ & $\begin{array}{c}4.45 \\
(3.1-10.1)\end{array}$ \\
\hline LDL-C/HDL-C & $\begin{array}{c}1.54 \\
(1.35-4.81)\end{array}$ & $\begin{array}{c}2.37 \\
(1.75-5.03) a\end{array}$ & $\begin{array}{c}2.47 \\
(1.78-3.53)^{a} \\
\end{array}$ & $\begin{array}{c}2.31 \\
(1.75-5.03)^{a}\end{array}$ \\
\hline TG/HDL-C & $\begin{array}{c}1.70 \\
(1.50-4.53)\end{array}$ & $\begin{array}{c}2.96 \\
(1.76-4.96) a\end{array}$ & $\begin{array}{c}2.88 \\
(1.66-5.18)^{\mathrm{a}} \\
\end{array}$ & $\begin{array}{c}3.17 \\
(2.16-4.96)^{a}\end{array}$ \\
\hline ApoA-I/apoB & $\begin{array}{c}2.10 \\
(1.79-2.25)\end{array}$ & $\begin{array}{c}1.49 \\
(1.13-2.03)^{a}\end{array}$ & $\begin{array}{c}1.49 \\
(1.14-2.09)^{\mathrm{a}} \\
\end{array}$ & $\begin{array}{c}1.46 \\
(1.10-1.73)^{\mathrm{a}}\end{array}$ \\
\hline hsCRP mg/L & $\begin{array}{c}1.50 \\
(0.16-3.5)\end{array}$ & $\begin{array}{c}3.68 \\
(0.16-49.5)^{a}\end{array}$ & $\begin{array}{c}3.11 \\
(0.16-49.5)^{\mathrm{a}} \\
\end{array}$ & $\begin{array}{c}4.26 \\
(1.28-17.6)^{a}\end{array}$ \\
\hline LPO $2 \mathrm{a} . \mathrm{m} . \mu \mathrm{Mol}$ & $\begin{array}{c}0.455 \\
(0.32-0.87) \\
\end{array}$ & $\begin{array}{c}1.02 \\
(0.48-1.53)^{a}\end{array}$ & $\begin{array}{c}1.05 \\
(0.54-1.53)^{\mathrm{a}} \\
\end{array}$ & $\begin{array}{c}1.00 \\
(0.48-1.38) a\end{array}$ \\
\hline LPO 7a.m. $\mu \mathrm{Mol}$ & $\begin{array}{c}0.442 \\
(0.28-0.83)\end{array}$ & $\begin{array}{c}0.75 \\
(0.60-0.96) a\end{array}$ & $\begin{array}{c}0.75 \\
(0.60-0.84)^{a} \\
\end{array}$ & $\begin{array}{c}0.75 \\
(0.690-0.96)\end{array}$ \\
\hline Melatonin 2a.m. ng/L & $\begin{array}{c}454 \\
(219-546) \\
\end{array}$ & $\begin{array}{c}432 \\
(161-510) \\
\end{array}$ & $\begin{array}{c}463 \\
(160-510) \\
\end{array}$ & $\begin{array}{c}362 \\
(165-470) \\
\end{array}$ \\
\hline Melatonin 7a.m. ng/L & $\begin{array}{c}485 \\
(205-519)\end{array}$ & $\begin{array}{c}382 \\
(226-503)^{a}\end{array}$ & $\begin{array}{c}418 \\
(238-503)\end{array}$ & $\begin{array}{c}342 \\
(226-472)\end{array}$ \\
\hline NT-proNBP ng/L & $\begin{array}{c}101 \\
(55-530)\end{array}$ & $\begin{array}{c}4243(1509- \\
35000)^{\mathrm{ab}}\end{array}$ & $\begin{array}{c}1910 \\
(1509-4331)^{a}\end{array}$ & $\begin{array}{c}7438(5014- \\
35000)^{\mathrm{ab}}\end{array}$ \\
\hline
\end{tabular}

a - vs. control group; b - vs. NYHA II

In patients with NYHA class II, Pearson's correlation test showed a significant negative correlation between the concentration of apoAI and LPO at 0:700 hrs $(r=-0.678$, $\mathrm{p}=0.016$ ), and a significant negative correlation between apoAII and LPO levels at 0:700 hrs $(\mathrm{r}=-0.581, \mathrm{p}=0.048)$. Moreover, multiple ridge stepwise regression showed a significant positive relationship between apoAII $\left(\mathrm{R}^{2}=0.730\right)$ and melatonin at $0: 200 \mathrm{hrs}(\beta=0.421, \mathrm{p}=0.046)$, and a significant negative relationship between apoAI $\left(\mathrm{R}^{2}=0.414\right)$ and LPO levels at 0:700 hrs $(\beta=-0.613, p=0.023)$. In CHF patients with NYHA class III, Pearson's correlation test showed a significant positive correlation between the concentration of apoAI and melatonin at 0:200 hrs $(\mathrm{r}=0.808, \mathrm{p}=0.008)$ and an insignificant positive correlation between apoAI and melatonin levels at $0: 700 \mathrm{hrs}(\mathrm{r}=0.658$, $\mathrm{p}=0.054)$. Furthermore, ApoAII was correlated significantly positively with melatonin at $0: 200 \mathrm{hrs}(\mathrm{r}=0.872, \mathrm{p}=0.002)$ and significantly positively correlated with melatonin at 0:700 hrs $(r=0.820, p=0.007)$ concentrations. However, hsCRP was significantly negatively correlated with melatonin at $0: 200 \mathrm{hrs}(\mathrm{r}=-0.834, \mathrm{p}=0.005)$ and significantly negatively correlated with melatonin at $0: 700 \mathrm{hrs}(\mathrm{r}=-0.719$, $\mathrm{p}=0.029$ ), as well as being significantly negatively correlated with apoAI $(\mathrm{r}=-0.693, \mathrm{p}=0.038)$ and significantly negatively with apoAII levels $(\mathrm{r}=-0.743, \mathrm{p}=0.022)$. Moreover, NT-proBNP was significantly negatively correlated with melatonin at $0: 700 \mathrm{hrs}(\mathrm{r}=-0.679, \mathrm{p}=0.044)$ concentration (Tab. 2). In CHF patients with NYHA class III, by way of multiple ridge stepwise regression, a significant positive relationship was noted between the concentration of apoAI $\left(\mathrm{R}^{2}=0.589\right)$ and melatonin at $0: 200 \mathrm{hrs}(\beta=0.735$, $\mathrm{p}=0.015)$, and a significant positive relationship was seen between apoAII $\left(\mathrm{R}^{2}=0.692\right)$ and melatonin levels at 0:200 hrs $(\beta=0.793, p=0.005)$, but a significant negative relationship was found between NT-proBNP $\left(\mathrm{R}^{2}=0.653\right)$ and melatonin at 7 a.m. $(\beta=-0.837, p=0.016)$ level, and an insignificant negative relationship was evident between $\operatorname{hsCRP}\left(\mathrm{R}^{2}=0.694\right)$ and melatonin at $0: 200 \mathrm{hrs}(\beta=-0.602$, $\mathrm{p}=0.058$ ) concentration (Tab. 3 ).

Table 2. Pearson's correlation between melatonin at 0:200 hrs and 0:700 hrs, NT-proBNP, hsCRP, LPO at 0:200 hrs and 0:700 hrs, and apoAI, apoAII, apoB - in patients with NYHA class III

\begin{tabular}{|l|c|c|c|c|}
\hline & $\begin{array}{c}\text { apoAI, } \mathrm{n}=10 \\
\mathrm{r}, \mathrm{p}\end{array}$ & $\begin{array}{c}\mathrm{apoAII}, \mathrm{n}=10 \\
\mathrm{r}, \mathrm{p}\end{array}$ & $\begin{array}{c}\text { NtproBNP, } \\
\mathrm{n}=10 \mathrm{r}, \mathrm{p}\end{array}$ & $\begin{array}{c}\text { hsCRP, } \mathrm{n}=10 \\
\mathrm{r}, \mathrm{p}\end{array}$ \\
\hline apoAI & & & -0.238 & $-0.693 *$ \\
\hline apoAII & 0.662 & & -0.516 & $-0.743 *$ \\
\hline Melatonin 2a.m. & $0.808 * *$ & $0.872 * *$ & -0.526 & $-0.834 * *$ \\
\hline Melatonin 7a.m. & 0.658 & $0.820 * *$ & $-0.679 *$ & $-0.719 *$ \\
\hline
\end{tabular}

* $-\mathrm{P}<0.05 ; * *-\mathrm{P}<0.01$

Table 3. Multiple ridge forward regression analysis between melatonin at 0:200 hrs and 0:700 hrs, NT-proBNP, hsCRP, LPO at 0:200 hrs and 0:700 hrs, and apoAI, apoAII, apoB - in patients with NYHA class III

\begin{tabular}{|l|c|c|c|c|}
\hline & $\begin{array}{c}\text { apoAI } \\
\mathrm{n}=10 \\
\mathrm{R} 2=0.589\end{array}$ & $\begin{array}{c}\text { apoAII } \\
\mathrm{n}=10 \\
\mathrm{R} 2=0.692\end{array}$ & $\begin{array}{c}\text { NTproBNP } \\
\mathrm{n}=10 \\
\mathrm{R} 2=0.653\end{array}$ & $\begin{array}{c}\mathrm{h} \text { (SCRP } \\
\mathrm{n}=10 \\
\mathrm{R} 2=0.694\end{array}$ \\
\hline Melatonin 0:200 hrs & $0.735^{*}$ & $0.793 * *$ & & -0.602 \\
\hline Melatonin 0:700 hrs & & & $-0.837 *$ & \\
\hline
\end{tabular}

$*-\mathrm{P}<0.05 ; * *-\mathrm{P}<0.01$

What is more, Pearson's correlation test (Tab. 4) in all CHF patients showed a significant positive correlation between concentration of apoAI and melatonin at 0:200 hrs $(\mathrm{r}=0.445, \mathrm{p}=0.049)$ and a significant positive correlation between apoAI and melatonin levels at 0:700 hrs $(r=0.461$, $\mathrm{p}=0.041)$. ApoAII was also correlated significantly positively with melatonin at 0:200 hrs $(\mathrm{r}=0.632, \mathrm{p}=0.003)$ and significantly positively correlated with melatonin at 0:700 hrs $(r=0.568, p=0.009)$ concentration. However, NT-proBNP was correlated significantly negatively with apoAI $(\mathrm{r}=-0.577, \mathrm{p}=0.008)$ and significantly negatively with apoAII $(r=-0.602, p=0.005)$ levels. In addition, Pearson's correlation test showed a significant negative correlation between hsCRP and melatonin at 0:200 hrs $(\mathrm{r}=-0.505$, $\mathrm{p}=0.023)$, and between hsCRP and melatonin at 0:700 hrs $(\mathrm{r}=-0.592, \mathrm{p}=0.006)$, as well as a significant negative relationship between hsCRP and apoAII $(\mathrm{r}=-0.471, \mathrm{p}=0.036)$ concentrations.

In all CHF patients, multiple ridge stepwise regression showed a significant positive relationship between the concentration of apoAII $\left(\mathrm{R}^{2}=0.879\right)$ and apoB $(\beta=0.341$, $\mathrm{p}=0.003)$, and between apoAII $\left(\mathrm{R}^{2}=0.879\right)$ and melatonin at $0: 200 \mathrm{hrs}(\beta=0.407, \mathrm{p}=0.0005)$, but a significant negative relationship between apoAII $\left(\mathrm{R}^{2}=0.879\right)$ and LPO levels at 0:700 hrs $(\beta=-0.268, p=0.009)$. However, an analysis of the concentration of hsCRP $\left(\mathrm{R}^{2}=0.465\right)$ showed a significant negative relationship with melatonin at 0:700 hrs $(\beta=-0.519, p=0.009)$, and NTproBNP $\left(R^{2}=0.656\right)$ revealed 
a significant negative relationship with apoAII $(\beta=-0.667$, $\mathrm{p}=0.014)$ level (Tab. 5).

Table 4. Pearson's correlation test between melatonin at 0:200 hrs and melatonin at 0:700 hrs, and NT-proBNP, hsCRP, LPO at 0:200 hrs and LPO at 0:700 hrs, and apoAI, apoAII, apoB - in all CHF patients

\begin{tabular}{|l|c|c|c|c|}
\hline & $\begin{array}{c}\text { apoAI, } \mathrm{n}=27 \\
\mathrm{r}, \mathrm{p}\end{array}$ & $\begin{array}{c}\text { apoAII, } \mathrm{n}=27 \\
\mathrm{r}, \mathrm{p}\end{array}$ & $\begin{array}{c}\text { NTproBNP } \\
\mathrm{n}=27, \mathrm{r}, \mathrm{p}\end{array}$ & $\begin{array}{c}\text { hsCRP, } \mathrm{n}=27 \\
\mathrm{r}, \mathrm{p}\end{array}$ \\
\hline apoAI & & & $-0.577 * *$ & -0.395 \\
\hline apoAII & $0.695 * * *$ & & $-0.602 * *$ & $-0.471 *$ \\
\hline Melatonin 0:200 hrs & $0.445 *$ & $0.632 * *$ & -0.204 & $-0.505 *$ \\
\hline Melatonin 0:700 hrs & $0.461 *$ & $0.568 * *$ & -0.377 & $-0.592 * *$ \\
\hline
\end{tabular}

Table 5. Multiple ridge forward regression analysis between melatonin at 0:200 hrs and 0:700 hrs, and NT-proBNP, hsCRP, LPO at 0:200 hrs, LPO at 0:700 hrs, and apoAI, apoAII, apoB - in all CHF patients

\begin{tabular}{|l|c|c|c|c|}
\hline & $\begin{array}{c}\text { apoAI, } \mathrm{n}=27 \\
\mathrm{R} 2=0.489\end{array}$ & $\begin{array}{c}\text { apoAII, } \mathrm{n}=27 \\
\mathrm{R} 2=0.879\end{array}$ & $\begin{array}{c}\text { NTproBNP, } \\
\mathrm{n}=27 \\
\mathrm{R} 2=0.656\end{array}$ & $\begin{array}{c}\text { hsCRP, } \mathrm{n}=27 \\
\mathrm{R} 2=0.465\end{array}$ \\
\hline apoAII & $0.492 *$ & & $-0.667 *$ & \\
\hline LPO 0:700 hrs & & $-0.268 * *$ & & \\
\hline apoB & & $0.341 * *$ & & \\
\hline Melatonin 0:200 hrs & & $0.407 * *$ & & \\
\hline Melatonin 0:700 hrs & & & & $-0.519 * *$ \\
\hline
\end{tabular}

\section{DISCUSSION}

Dyslipidemia and the resulting atherosclerosis as a chronic disease remains one of the major causes of death, worldwide. Dyslipidemia and dyslipoproteinemia play a crucial role in cardiovascular diseases through the development of atherosclerosis and the modification of the structure and stability of the cellular membranes [2,3]. Melatonin has anti-inflammatory and anti-oxidative effects, neutralizes free radicals, increases antioxidative enzymes and glutathione levels, prevents electron leakage from the mitochondrial respiratory chain, acts synergistically with vitamins $\mathrm{C}, \mathrm{E}$, and glutathione, reduces levels of pro-inflammatory cytokines, and, therefore, prevents low-density lipoprotein (LDL) oxidation and decreases lipid peroxidation $[1,7,16]$. The results of human and animal studies support the consideration of melatonin as a cardio-protective agent $[1,4,5,14,15,18,20]$. In addition, melatonin demonstrates a lowering impact on blood pressure, the normalization of lipid profile, and certain anti-inflammatory properties [4,16,23,24]. Recently, Tamura H. et al. [22] has reported that melatonin treatment in periand postmenopausal women elevates serum high density lipoprotein cholesterol levels without influencing total cholesterol levels [22]. However, the mechanisms of beneficial effect of melatonin on these processes remain unknown.

The presented study concerned observations of melatonin and HDL metabolism linkage in all patients with CHF, and with subgroups with NYHA class II and III. CHF patients without acute inflammatory response demonstrated dyslipidemia and dyslipoproteinemia which was characterized by lowered concentrations of HDL-C, apoAI, apoAII. While NT-proBNP, hsCRP and LPO levels at night and during daytime were elevated, the melatonin level did not show such elevation. Moreover, in all CHF patients, as well as in patients with NYHA class II and III, the concentration of LPO at 0:700 hrs was lower than at 0:200 hrs. In addition, a significant negative Pearson's correlation between concentration of apoAI and LPO at 0:700 hrs, and between apoAII and LPO at 0:700 hrs was observed in the group of patients with NYHA class II. However, to have more relevant and essential correlations, multiple ridge stepwise forward regression has been performed. Hence, multiple ridge stepwise regression showed a significant positive relationship between concentration of apoAII and melatonin at 0:200 hrs, but a significant negative relationship between apoAI and LPO levels at 0:700 hrs. These results indicate that melatonin at night exerts a positive effect on apoAII level, and the content of melatonin at 0:200 hrs, apoAI and apoAII, together, have an influence on lowered oxidative stress in CHF patients with NYHA class II.

In this work, Pearson's correlation test and multiple ridge stepwise regression in CHF patients with NYHA III showed a significant positive relationship between concentration of apoAI and melatonin at 0:200 hrs, and between apoAII and melatonin at 0:200 hrs. However, negative relationships were noted between the hsCRP and melatonin (0:200 hrs) and between NT-proBNP and melatonin (0:700 hrs). Moreover, the obtained results suggest that melatonin measured at 0:200 hrs exerts a positive effect on apoAI and apoAII content and HDL composition, which altogether influence the lowering of NT-proBNP during daytime, in patients with advanced CHF without acute inflammation.

In addition, Pearson's correlation test and multiple ridge stepwise regression in all CHF patients showed a significant positive relationship between concentration of apoAII and melatonin at 0:200 hrs, and between apoAII and apoB. Furthermore, a significant negative relationship between concentration of: apoAII and LPO at 0:700 hrs, and between apoAII and NT-proBNP levels, as well as melatonin (0:700 hrs) and hsCRP were noticed. This in turn indicates that melatonin at night may have an influence on rising apoAII, and thus causes a decrease in LPO at 0:700 hrs, as well as NT-proBNP levels in all CHF patients.

The presented results have been confirmed by other researchers [14], who reported that melatonin clearly slowed lipid peroxidation and apoB carbonylation in LDL, together with a concomitant protection of LDL endogenous antioxidants, i.e., $\alpha$-tocopherol and $\beta$-carotene. Korkmaz A. et al. [12] revealed that the results of human and animal studies support the consideration of melatonin as a cardio-protective agent. These findings are virtually consistent with melatonin being beneficial for cardiovascular physiology, and in the reduction of free radical damage to the myocardium. Considering its low toxicity, melatonin deserves consideration for inclusion in clinical trials attempting to identify molecules with cardiovascular benefits [12].

Of note, the high anti-oxidant and anti-inflammatory activities of HDL are associated with protection against cardiovascular disease. These activities depend on the protein and lipid composition of HDL and are the greatest in small, dense HDL $[10,11,19]$. These activities are compromised in many pathological states related with inflammation. In 
addition, the functional deficiency of HDL is intimately associated with changes in the composition of HDL. Research is, thus, being continued to find the best approaches for prevention of the loss or restoration of the antioxidant and anti-inflammatory potential of HDL $[2,10,11,19,21]$. Of further note is that the structure and metabolism of HDL are linked to their apoAI and apoAII composition; however, the role of apoAII in the metabolism of HDL is unknown. What is known is that conformational changes of apoAI, the major apolipoprotein of HDL, caused by apoAII in discoidal HDL are confined to two regions of apoAI. In addition, interactions between the two major apolipoproteins in discoidal HDL are site specific. Functional implications of HDL complexes will significantly benefit from such structural information [9]. Of still further note, Apo A-II participates in ABCA1-mediated HDL formation, whereas nascent HDL maturation by LCAT is associated with apo A-I, apo C-I and apo A-IV [13]. Apolipoprotein A-II is a key regulatory factor of HDL metabolism as evidenced from studies with transgenic animals and from clinical outcomes. What is more, HDL metabolism and functionality are closely linked to their major apolipoproteins A-I and A-II, and to certain cargo proteins. However, beyond HDL cholesterol concentrations are needed to identify the discrete HDL particles that are atheroprotective [13]. Recent evidence suggests that the cardioprotective effect of the HDL system may relate chiefly to both the apoAI and apoAII content of HDL particles [17]. A prospective study demonstrated that plasma apoA-II concentrations are an independent factor negatively correlated with the risk of coronary artery disease (CAD) [3]. This challenges the previous notion that apoAII is proatherogenic $[3,17]$. Dzida et al. [6] showed in their preliminary results that in patients with advanced CHF (NYHA class III but not NYHA class II), nocturnal melatonin secretion is negatively correlated with NT-proBNP, and suggests that reduced melatonin concentration may result from melatonin synthesis inhibition due to neurohormonal activation [6]. The presented study demonstrates, for the first time, the beneficial effect of melatonin on apoAII and on apoAI composition of HDL particles, and that all these elements are closely related with the functionality of HDL. The presented study also reveals that nocturnal melatonin beneficially changes the content of apoAI and apoAII of HDL particles and the antioxidative, anti-inflammatory function of HDL, but does not have such an influence on NT-proBNP level in patients with NYHA class II. However, in patients with advanced CHF (NYHA class III), nocturnal melatonin has an impact on the increased content of apoAII and apoAI, while these apolipopoteins lower oxidative stress, inflammation, and, together with melatonin, at daytime, decrease the NT-proBNP level.

\section{CONCLUSION}

The presented results indicate that nocturnal melatonin exerts a positive effect on the composition of HDL particles which are smaller and denser in patients with CHF. The content of apoAII and apoAI in HDL particles and melatonin level appear to have anti-oxidative and anti-inflammatory effects, and altogether have a beneficial cardio-protective effects in patients with advanced CHF. These relationships, however, need to be confirmed in further studies.

\section{REFERENCES}

1. Aqil A., Reiter R.J., Jimenez-Aranda A., Iban-Arias R., NavarroAlarcon M., Adem A., Fernandez-Vazquez G. Melatonin ameliorates low-grade inflammation and oxidative stress in young Zucker diabetic fatty rats. J. Pineal Res., 54, 381, 2013.

2. Berrougui H., Claudia N. Momo CN., Khalil A.: Health benefits of high-density lipoproteins in preventing cardiovascular diseases. J. Clin. Lipidol. 6, 524, 2012.

3. Birjmohun R.S., Dallinga-Thie G.M., Kuivenhoven J.A., et al.: Apolipoprotein A-II is inversely associated with risk of future coronary artery disease. Circulation, 116, 2029, 2007.

4. Dominguez-Rodriguez A.: Melatonin in cardiovascular disease. Expert. Opin. Investig., 21, 1593, 2012.

5. Dominguez-Rodriguez A., Abreu-Gonzalez P., Avanzas P.: The role of melatonin in acute myocardial infarction. Front. Biosci., 17, 2433, 2012.

6. Dzida G., Prystupa A., Lachowska-Kotowska P., Kardas T., Kamieński P., Kimak E., Hałabiś M., Kiciński P.: Alteration in diurnal and nocturnal melatonin serum level in patients with chronic heart failure. AAEM., 20, 745, 2013.

7. Espino J., Pariente JA., Rodriguez A.B.: Oxidative stress and immunosenescence: therapeutic effects of melatonin. Oxidative Med. Cell. Longev., 2012, 6702941, 9p, 2012.

8. Friedewald W.T., Levy R.I., Friedrickson D.S., Estimation of the concentration of low-density lipoprotein cholesterol in plasma without the use of the preparative ultracentrifuge. Clin. Chem., 18, 499, 1972.

9. Gauthamadasa K., Vaitinadin N.S., Dressman J.L., Macha S., Homan R., Greis K.D., Silva G.D.: Apolipoprotein AII-mediated conformation changes of apolipoprotein AI in discoidal high density lipoproteins. J. Biol. Chem., 287, 7615, 2012.

10. Kimak E., Bylina J., Solski J., Hałabiś M., Baranowicz-Gąszczyk I., Książek A. Association between lipids, lipoproteins composition of HDL particles and triglyceride-rich lipoproteins, and LCAT and CETP activity in post-renal transplant patients. CBB., 67, 695, 2013.

11. Kimak E., Hałabiś M., Baranowicz-Gąszczyk I., Solski J., Książek A.: Association between moderately oxidized low-density lipoprotein and high-density lipoprotein particle subclass distribution in hemodialyzed and post-renal transplant patients. JZUS-B., 12, 365, 2011.

12. Korkmaz A., Reiter R.J., Topal T., Manchester L.C., Oter S., Tan D.X.: Melatonin, an established antioxidant worthy of use in clinical trials. Mol. Med., 15, 43, 2009.

13. Maïga S.F., Kalopissis A-D., Chabert M.: Apolipoprotein AII is a key regulatory factor of $\mathrm{HDL}$ metabolism as appears from studies with transgenic animals and clinical outcomes. Biochimie., 96, 56, 2014.

14. Marchetti C., Sidahmed-Adrar N., Collin F., Jore D., Garde's-Albert M., Bonnefont-Rousselot D.: Melatonin protects PLPC liposomes and LDL towards radical-induced oxidation. J. Pineal. Res., 51, 286, 2011.

15. Morceli G., Honorio-Franca A.C., Fagundes D.L., Calderon I.M., Franca E.L.: Antioxidant effect of melatonin on the functional activity of colostral phagocytes in diabetic women. PloSOne 8, e56915. Epub 2013.

16. Mozaffari S., Hasani-Ranjbar S., Abdollahi M.: The mechanisms of positive effects of melatonin in dyslipidemia: a systemic review of animal and human studies. Int. J. Pharmacol., 8, 496, 2012.

17. Ng T.W.K., Chan D.C., Hugh P., Barrett R., Watts G.F.: Effect of weight loss on HDL-apoAII kinetics in the metabolic syndrome. Clin. Sci., 118, 79, 2010.

18. Papaharlambus C.A, Griendling K.K.: Basic mechanisms of oxidative stress and reactive oxygen species in cardiovascular injury. Trends Cardiovasc. Med., 17, 48, 2007.

19. Podrez E. A.: Anti-oxidant properties of high-density lipoprotein and atherosclerosis. Clin. Exp. Pharmacol. Physiol., 37, 719, 2010.

20. Reiter R. J., Tan D-X., Paredes S.D.: Beneficial effects of melatonin in cardiovascular disease. Ann. Med., 42, 276, 2010. 
21. Shuhei N., Söderlund S., Jauhiainen M., Taskinen M-R.: Effect of HDL composition and particle size on the resistance of HDL to the oxidation. Lipid Health Dis., 9, 104, 2010.

22. Tamura H., Nakamura Y., Narimatsu A., Yamagata Y., Takasaki A., Reiter R.J.: Melatonin treatment in peri- and postmenopausal women elevates serum high density lipoprotein cholesterol levels without influencing total cholesterol levels. J. Pineal. Res., 45, 101, 2008.
23. Wakatsuki A., Okatani Y., Ikenoue N., Shinohara K., Watanabe K., Fukaya T.: Melatonin protects against oxidized low-density lipoprotein-induced inhibition of nitric oxide production in human umbilical artery. J. Pineal Res., 3,: 281, 2001.

24. Wu W.S., Chou M.T., Chao C.M., Chang C.K., Lin M.T., Chang C.P.: Melatonin reduces acute lung inflammation, edema, and hemorrhage in heatstroke rats. Acta Pharmacol. Sin., 33, 775, 2012. 